\title{
Memory-based and on-line processing of metaphoric sarcasm
}

\begin{abstract}
Metaphoric statements such as "He certainly is Romeo to her Juliet" can be uttered not only as a metaphor but used sarcastically to make a commentary on the metaphor. So how does one determine the intent of the speaker who utters such a statement? Evidence is reviewed about the constraints that are available for use by someone who reads or hears such a statement and an experiment is described which indicates that at least some of these constraints work on-line, that is during the moment-to-moment processing of the statement as it is encountered.
\end{abstract}

There is an inherent ambiguity in interpreting what is intended by certain forms of language. Consider statements such as "He certainly is Romeo to her Juliet" (1) or "He certainly is Abelard to her Heloise" (2). When, in fact, would someone make such a statement and what could someone who hears (or reads) it, take it to mean? The ambiguity here can arise from at least two sources: the familiarity of the items (in this instance, people) being compared and the message intended by the comparison.

Consider for instance the role that familiarity might play. Most students of Western culture would be familiar with the Shakespearean story of Romeo and Juliet and so, on one level, the metaphoric comparison of (1) would be understandable: He (the person being described) is being compared to Romeo, son of the Montague, the lover of Juliet, daughter of Capulet, the arch enemy of the Montague clan. Indeed, one could argue that "Romeo and Juliet" might serve as a prototype for certain types of romantic relationships and that the interpretation of the statement would arise by classifying the topic $(\mathrm{He})$ to the category suggested by the nominal vehicle represented by "Romeo and Juliet" (see, for instance, 
Glucksberg, 1991). That is, one could see the statement as a metaphor in which the person being described is depicted in a passionate but tragically doomed relationship.

Understanding (2) would not be as easy because the story of Abelard and Heloise is not as well known. In fact, without the requisite background knowledge the statement would be non-transparent and any one of a number of possible relationships might be plausible. Naturally, the same problem arises for (1), for those people who are ignorant of the Shakespearean story. The point here is that the meaning of the statement is dependent upon stored background knowledge.

Without such knowledge one is left in making inferences based on the grammatical information alone. If one were to assume that " $\mathrm{X}$ (a person) certainly is $\mathrm{Y}$ (a person) to her Z (a person)" (3), acts as a construction in construction grammar terms (see Kay, 1997) then some additional (albeit limited) information could, arguably, be made available. For instance, one might tend to use that construction to describe a limited number of types of interpersonal relationships with the exemplar being a prototype of each type. But, in any event, limiting our investigation to key decontextualized statements, as is so often done in the metaphor (and indeed, in general, the psycholinguistic) literature, inevitably produces an impoverished environment for inferring speaker's intent.

In real-life processing of language we do not encounter statements such as (1) or (2) out-of-context (see Katz, 1996a). Language is inextricably contextual and the contexts are both rich and complicated. One should not think of context in a simple unidimensional manner but rather as a set of embedded linguistic and extralinguistic constraints, some of the latter being nonverbal or gestural in nature (see, for instance. Kelly, Barr, Church \& Lynch, 1999). The contextual constraints that are linguistic in nature include, but are not limited to: restrictions on the number of plausible continuations invited by the discourse in which the statement is embedded (see, for instance, $\mathrm{Vu}$, Kellas, \& Paul, in press); the pragmatic implications to speakers intent created by markers, such as the use of terms such as "really" or "truly" or even use of a simile versus a nonsimile contrast; sensitivity to the conditions in which one would use hyperbole or understatement (see, for instance, Kreuz, 1996), or variations in prosody (see, for instance, McDonald, 2000). In addition to constraints invited by the use of gestures, other extra-linguistic contextual constraints include stored knowledge about the social or power relationships among the persons in the conversation (see, for instance, Holtgraves, 1994; Kreuz, 1996) or background knowledge about what we believe that other people know (what some label 'theory of mind'). This factor might be especially important for interpreting sarcasm and some other forms of indirect speech. For instance, consider the scenario in which three people are in a conversation, but only two of these know that a friend (John) has separated from another friend, Mary. If one of the people "in the know" states: "John and 
Mary are acting like love birds these days" (4), the other two people would inevitably draw different interpretations. Indeed the speaker might have framed the message in the way that he did on purpose: namely to send one message to the person in the know (who should recognize the irony intended by the speaker) and another message to the unaware communicant-a message intended to keep that person ignorant of the facts.

It is exactly these types of everyday contextual constraints that are essential for understanding what one is intending to convey by statements such as (1) or (2). If one were to base their understanding just on the bare statement itself one could, arguably, come up with a metaphoric interpretation. As noted above, often even this depends upon additional background knowledge. However, even if one were to make the argument that the metaphoric interpretation is the default, it clearly is not the only plausible meaning being intended. And here it is where contextual constraints come into play. Consider, for instance, the following simple conversation.

Speaker A: John can't stand Mary You can't even have them in the same room anymore.

Speaker B: Yeah. He certainly is Romeo to her Juliet.

Here it is obvious that Speaker B is using the metaphor to be ironic, or even, sarcastic. That is, understanding the comment requires knowledge both of the metaphor and the ironic spin made about it. Given the familiarity of the metaphoric base, the ironic spin should be quite transparent. Consider next the analogous conversation.

Speaker A: John can't stand Mary You can't even have them in the same room anymore. Speaker B: Yeah. He certainly is Abelard to her Heloise.

Here the situation is somewhat different since the ironic spin is not apparent, given the relative unfamiliarity of the contrast. One could doubtless understand Speaker B: the syntax is not ambiguous and, assuming that Gricean principles are being followed, one can assume that Speaker B was following conversational maxims. But what should Speaker A make of the comment, given ignorance of the Abelard-Heloise story? Assuming a metaphoric default, it is quite possible that the ironic intent is lost completely with Speaker A assuming that the example provided is of two people who typify an estranged couple.

The informal examples above are meant to illustrate the importance of two factors that go into interpreting metaphor: the familiarity of the item, and the nature of the context which constrains our understanding of the metaphor. In the following sections, I will, first, briefly examine a limited number of contextual 
constraints that can turn a metaphor comparison into an ironic commentary and, second, discuss whether these constraints come into play early in the interpretation process.

\section{Social Constraints and Metaphoric Irony}

As noted above, whether statements such as (1) or (2) are understood as metaphor or as ironic commentary depends on the context in which they are produced. An analogous point can be made of virtually any sentence that can be taken in a nonliteral way, even for familiar or canonical metaphors. (Consider, for instance. Someone saying: "My car is a lemon" in the context of a parade, in which the floats represent different types of fruit). What might be especially important for interpreting the metaphor as sarcasm is social knowledge, given that sarcasm involves a human being who is the "victim" of the verbal barb (see, for instance, Lee and Katz, 1998, wherein we demonstrated that sarcasm, but not irony, involves ridicule of a specific person or group of people). That is, understanding sarcastic statements would depend on contextual constrains that call upon knowledge of social convention and expectation. Indeed Ellen Winner (1988) makes the point that a child learns to recognize metaphor at an earlier age than he or she recognizes irony due to the fact that understanding social conventions (upon which irony is based) develops later than the cognitive skills required to understand metaphor.

Given the importance of context in general, and social factors in particular, one might think that extensive pretesting of the discourse contexts would occur to ensure the adequacy of the stimuli employed in experimental examinations of metaphor or irony processing. For the most part, however, experimenters appear to construct stimuli on an ad hoc basis, without careful consideration of the conditions under which a person might be expected to use certain linguistic constructions in everyday settings, though of course there are notable exceptions to this criticism (eg., Jorgensen, 1996). The point is here that the experimental stimuli should reflect real-life contextual constraints. A recent paper by Gibbs (2000) reinforces the need for such care. Gibbs analyzed the conversation of university students and their friends for instances of ironic speech and noted that five distinct types of irony were frequently employed: teasing jocularity, sarcasm, rhetorical questions, hyperbole and understatement. Moreover, social factors were found to be important: some types were more frequently used by males, others by females; and, most important, use of irony was often a collaborative social event, with an ironic comment being followed by ironic responses by others in the conversation. Presumably, to create experimental situations that simulate real- life conditions (and hence the contextual constraints that would be active in 
real-life language production and comprehension) at the least experimenters should construct discourse contexts that reflect the social conditions in which various forms of language are employed. To date, this care has not generally been observed, and, in fact, the even more modest attempt to ensure that the experimental stimuli are realistic is not the norm. Consider, for example, a study Maggie Toplak and I conducted to examine the reasons that a person might use an indirect sarcastic statement rather than the direct criticism. We employed items taken from a seminal paper on irony processing (Kreuz and Glucksberg, 1989), but, additionally, normed these items to see if our sample could imagine themselves in the scenario being described, and whether, in that scenario, they would have used the statement attributed to the speaker (Toplak and Katz, 2000).To our surprise, only about $1 / 3$ of the items from that seminal paper met our relatively modest attempt at ensuring ecological realistic discourse contexts. Clearly much more work is required in the experimental preparation of the stimuli we use to examine selected theoretical issues in the processing of language, especially non-literal language.

Given the caveat that extensive, comprehensive, and careful examination of sociallybased contextual constraints has yet to be done, I will describe two such social constraints that have already been identified: the nature of the relationship held by different people in communication, and the role played by social roles, such as those of gender and class.

\section{Relationships among the people in communication}

There is growing literature which points to the importance of systematically examining the role played by different participants in a conversation. For instance, the use of sarcasm has been shown to vary as a function of the degree of relatedness of the speaker and the "victim" of the speaker's barb (Jorgensen, 1996; Kreuz, 1996). Toplak and Katz (2000) have shown that different members in communication give somewhat different reasons why someone would make an indirect sarcastic statement. And, Kreuz, Kassler, Coppenrath and Allen (in press) have shown that the appropriateness of an ironic expression is associated with the amount of shared information between speaker and listener.

Katz and Lee (1993) manipulated contextual information based on whether or not members of a conversation shared background information (by virtue of being mutually present when some information was presented) or not (when the requisite information would be presented when one member of the conversational group was not present). The manipulation occurred by varying the nature of various texts, which our respondents were asked to rate: participants were presented a set of scenarios in which one person echoed an earlier statement 
made by someone else, with the echoic mention now being metaphoric in use. There were two manipulations of importance here: (1) whether the context would lead a listener to believe that the speaker was endorsing the statement or was being pragmatically insincere and (2) whether the listener was present or not when the original mention of the statement had been made. This last manipulation would lead to two groups of listeners: a privileged audience who would share with the speaker the knowledge of the context in which the statement had been made originally and a non-privileged audience which would not have access to this background contextual knowledge.

The findings, based on ratings given to authorial intent and degree of metaphoricity or ironicity, were very clear. If a listener thought the speaker was endorsing the truth-value of the statement then the comment was taken as a metaphoric description whereas, if the listener thought the speaker was actually rejecting the truth-value of the statement, it was taken as an ironic comment. Moreover, perception of the statement as being ironic varied as a function of audience privilege: an interpretation of irony was especially salient when the listener not only perceived a speaker to be making an incongruent (i.e., a rejecting) statement but also recognized that someone else in the conversation (the non- privileged listener) was unaware that the speaker was not in fact endorsing the position he or she just espoused.

\section{The Impact of Social Roles}

The variables noted above could be thought of a contextual constraints engendered by the social dynamics of communicating with people of varying degrees of relatedness and shared knowledge. In addition, there are contextual constraints engendered by social convention. That is, one could argue that there are social conventions associated with gender, socio-economic class, and power or status inequalities which could be used to disambiguate speaker's intent. An example of socio-economic factors is evident in the use of indirect requests, such as when one asks: "Can you open the window?"; taken literally this is a question about ability but pragmatically is understood as a request to perform the act. Holtgraves (1994) has shown that social status plays a role in the speed with which such statements are understood. Other research (Holtgraves, 1997a; Kemper and Thissen, 1981) has shown similar effects on our memory for statements differing in politeness: participants were more likely to remember statement form for wordings incongruous with social status, such as when a low status person talks in an impolite manner to a high status person. Analogous effects can be found with gender: female participants are more likely than males to interpret statements as being indirect (e.g., Holtgraves, 1991) and, as a function of power relationships, 
closeness of relationship with the person to whom she or he is talking and the size of the request being made, differ from males in their use of polite language (Holtgraves, 1992).

Taken together these data can be taken as strong evidence for socially driven interpersonal factors in interpreting somewhat ambiguous statements. Penny Pexman and I asked whether we could demonstrate another socially-driven effects in interpreting sarcastic metaphor (Katz and Pexman, 1997). Our study was quite simple. We were interested to see whether a social factor - in our case, the occupation of a speaker influenced the likelihood that a statement that could be understood as metaphor or as a sarcastic commentary. Moreover, we were interested to see whether any such effects would be moderated by the discourse context or the familiarity of the statement. To conduct this study we first had to demonstrate a difference in the perceptions of language usage for members of different occupations. We obtained norms that clearly showed some differences: clergymen, doctors, and teachers were perceived as likely to use metaphor (and less likely to use irony) in everyday speech whereas comedians, police and factory workers were perceived as more likely to use irony (but not metaphor) (in a related manner see Holtgraves, 1997b for evidence of cross cultural and individual differences in using indirect language).

Once we obtained these occupational differences, we constructed scenarios. In one scenario a statement in the form of a nominal metaphor (e.g., "children are precious gems") was placed in a discourse that either brought out the metaphoric sense or an ironic usage or was neutral with respect to the metaphor vs. ironic difference. The nominal metaphors were either highly familiar instances of basic conceptual metaphors or were novel instances. For each of the three contexts, the nominal metaphor would be uttered by a person who was perceived as high metaphor users (e.g., clergyman) or high irony users (e.g., comedian). A fourth control condition was also constructed: this was the same as the neutral condition described above with the difference that the speaker was not associated with any specific occupation. Naturally, passages were counterbalanced such that any one person only saw the target sentence in one context, uttered by a person from one occupation but, across the study, all key passages were uttered by someone from each occupation (or, in the final control condition, by a person from no specified occupation).

Two types of dependent measures were taken. First, after each scenario, participants answered a set of questions regarding the intent of the speaker, two of which were especially relevant, namely whether the speaker was sarcastic and whether the speaker was mocking someone else. Across studies we manipulated, in the rating questions themselves, whether or not in the ratings we made salient the occupation of the speaker. Second, after all the scenarios were rated and after completing a distraction task, participants were asked to recall as many of the target sentences as possible. 
The results were very clear With respect to the rating data, the discourse contexts acted as one would expect with the key statement perceived as more sarcastic when placed in a counterfactual context meant to bring out the ironic commentary. There was no difference between the neutral context and the context meant to bring out the metaphoric sense, suggesting that the default was to treat the comparison as a metaphor. Of greater interest was the influence of speaker occupation. When compared to the neutral conditions (the two in which speaker occupation was mentioned and the one in which it was not), speakers from high- irony occupations were perceived as more sarcastic, at least for the highly familiar statements. When the occupation was made salient in the rating scale, the effects were even more general: statements made by speakers of high-irony occupations were seen as being more sarcastic than the exact same statements made by high- metaphor speakers. Overall then, familiar statements were perceived as especially sarcastic when uttered in a irony-inducing context or when uttered by a member of a high-irony occupation. The memory data confirmed the intluence of speaker occupation: statements uttered by members of high-irony occupations were better recalled than the same statements made by members of highmetaphor occupations. Since the statements and contexts were identical in every other way, these data suggest that occupation was coded somewhere in the process of comprehension.

Taken together, these data clearly indicate that the social role of the person who makes a statement plays an important role in our perception of a given statement as being a metaphor or a sarcastic commentary on that metaphor. Moreover, these effects interact with the familiarity of the statement itself Our interpretation of these data was that the metaphoric content of a familiar statement would be available for use, regardless of context. Thus when the context was consistent with the metaphoric interpretation, one need not look for an alternative (ironic) usage. However when the context is non-endorsing and suggests a sarcastic usage, the incongruity between the familiar metaphoric use and the ironic context is made especially salient, leading to a heightened sense of sarcasm. Incongruity is less marked with unfamiliar statements because those statements are not associated with metaphoric use. An analogous argument is made with respect to speaker occupation: in the neutral condition participants have little explanation of why the speaker made the comment. Participants provided with highly familiar statements know that the statement is likely to be used as a metaphor and it is made by a person associated with either metaphoric or ironic speech. Only in the latter case does a sense of incongruity obtain, and is reflected in the sarcasm ratings. With unfamiliar items however, the metaphoric content of the statement is much less salient, and the sense of incongruity does not arise.

Taken together these data provide evidence that people are sensitive to factors such as the social roles of a speaker, and that the influence of these roles interacts 
with constraints engendered by the discourse context and the salience of the statement itself. The interactive effects of speaker occupation, discourse context, and statement familiarity suggests that multiple sources of information are been weighed when one attempts to understand the intent behind the use of a given statement.

\section{On-line processing and the Role of Contextual Constraints}

Above, we have made the argument for the importance of social knowledge as contextual constraints in determining the metaphoric or sarcastic intent of certain ambiguous statements. The question of interest here is when do these contextual factors come into play? For instance, do the constraints work very early in the processing sequence so as to limit the number of plausible interpretations to the ambiguous statement, or, alternatively, do the contextual constraints come into play relatively late, after other cognitive analyses have already been completed. This issue has been quite controversial in the literature with the classic approach being the latter (e.g. Searle, 1979): the standard position here being that one is obligated to initially process for the literal sense of a trope and only when that fails to provided a contextually adequate interpretation does one then go on and try to find a plausible (and nonliteral) interpretation. In contrast, there is by now a twenty-year old experimentally based position, and the currently accepted received wisdom, which claims that, with appropriate contextual support, the nonliteral meaning is made available as rapidly as the literal sense and that the access of the nonliteral interpretation is not dependent on a failure to achieve a plausible literal interpretation occurs (see Katz, 1996b for a review). There is some indication that the received wisdom has been best demonstrated with familiar tropes and that less familiar nonliteral meaning might still involve the initial processing of either literal meaning (see Turner and Katz, 1997) or what Rachel Giora labels salient meaning, that is, coded meaning such as occurs when the nonliteral sense is the most familiar usage of the item (see Giora, Fein \& Schwartz, 1998). This alternative view would hold that contextual constraints that limit the meaning of a target sentence would be present when those sentences are used in their dominant sense; however, for novel uses or non-dominant or non-salient meaning, the literal meaning would have processing priority and the less dominant meaning would only emerge later in the processing sequence.

The contrast between whether contextual constraints are primary and limit what interpretations occur to some verbal information during the act of reading that information or whether contextual constrains come into play after the fact is relevant not only to our understanding of sarcasm comprehension per se, but to a larger set of issues in language processing. This controversy can he found 
from "low-level" activities such as at the level of word access (e.g, Vu et al, in press) and resolution of syntactic ambiguities (e.g., McRae, Spivey-Knowlton \& Tanenhaus, 1998), all the way to "high-level" activities such as the processing of metaphor (see Glucksberg, 1991), sarcasm (Giora et al., 1998) and proverbial language (e.g., Turner and Katz, 1997).

Examination of these issues require on-line studies of the reading process, that is, measures that are sensitive to the moment to moment processing that occurs as one reads or hears the critical information. For instance, consider again statements such as (1), read in the following contexts. "John and Mary really seem to hate each other. You can't even leave them alone in the same room anymore. You can say that he is certainly Romeo to her Juliet" versus "John and Mary really seem to love each other. You can't even leave them alone in the same room anymore. You can say that he is certainly Romeo to her Juliet". Assuming familiarity with the contrast, the former usage is ironic whereas the latter one is metaphoric. We have discussed how familiarity, the nature of the discourse context and social factors, such as the person who would make such a comment all play a role in helping us come to the realization of ironic intent. The questions of interest are, first, whether the ironic sense would emerge as rapidly as the metaphoric sense if the context was such that discourse and social factors were both consistent with either the metaphoric or ironic usage and, second, whether there is evidence that the metaphoric or ironic meaning arises very early, during the processing of target items, such as (1), or whether the meaning arises more slowly, sometime after the target is no longer being read or heard.

The data on this issue are somewhat inconclusive. Most of the data reported above which indicated a role in disambiguating the speaker's intent (such as that based on knowledge of the speaker's occupation or gender) is memory-based (i.e., dependent on recall measures directly, or those based on one's memory, such as ratings taken some time after an item had been processed) and not online in nature. There are a limited number of relevant on-line studies. Blasko and Connine (1993) for instance, using a cross-modal priming procedure, demonstrated that the nonliteral meaning of familiar and uf apt metaphors is accessed early in the processing sequence, and, using measures of brain activity, Pynte, Besson, Robichon and Poli (1996) provide evidence that they take to indicate that, when contextually relevant, the metaphorical meaning of a sentence is the only one accessed. Both of these studies are consistent with the received wisdom that, when contextually supported, the metaphoric meaning is processed early in the seduence and hence unlikely to be dependent on a prior (and obligatory) processing of literal meaning.

The evidence with sarcastic statements is less clear: Gibbs (1986) presented evidence that the sarcastic sense is processed as rapidly as the literal sense (and 
hence occurs early in the processing sequence and is constrained by context) whereas, supporting her graded saliency position, Giora provides evidence that the ironic sense of a statement takes longer to develop than does the literal sense (and hence occurs later in processing and, for non-salient items, context does not constrain which meaning is accessed-see Giora et al., 1998). Although these studies are both well executed, neither was constructed with the richness of contextual constraints in mind. Recall the argument made earlier that a multiplicity of contextual constraints are in principle available for use, and that social factors are especially important in inviting an ironic interpretation of an ambiguous statement. As such, the differences between Gibbs and Giora et al may merely represent differences in the contextual constraints available: perhaps some constraints are more likely to work on-line whereas others are more inferential in nature and only work later in the processing sequence.

As a first step at examining the role of social contextual constraints, Penny Pexman, Todd Ferretti and I (Pexman, Ferretti and Karz, 2000) have recently completed an on-line study which examined the role of the three constraints examined in Katz and Pexman (1997). Recall that in the earlier study, we presented target items in the form of a nominal metaphor, namely "An A is (does) B". These items were either familiar or unfamiliar instantiations of basic conceptual metaphors, and were always presented in a short passage, being uttered by one of the characters in the story. Thus we examined the role that expressed familiarity played on disambiguating the meaning intended by the character who uttered the statement. A second constraint was the nature of the discourse that preceded the target item: the discourse either suggested the target statement was an endorsement and hence being used as a metaphor (the metaphor-inviting condition) or suggested that the speaker was rejecting the truth-value of the statement and hence using the metaphor to make a sarcastic commentary (the sarcasm-inviting condition) or was neutral with respect to endorsement-rejection. Finally, we manipulated a social factor, namely the occupation of the person who made the statemem (either high-irony speaker, high-metaphor speaker, or a control condition in which speakers occupation was not presented). The findings of this earlier study was clear using memory-based dependent measures (either recall or ratings of sarcasm and mocking): each of these constraints helped to disambiguate the metaphoric or sarcastic intent of the speaker, otten acting interactively.

Our interest was to see whether any of these three constraints acted on-line, during the reading of the target item, or whether effects only occurred later in the processing sequence. Our task employed a self paced moving window procedure in which readers advance a text word-by-word across a computer screen at their own pace. This procedure mimics the eye movements found in normal reading, removes spurious strategies adopted by readers forced to read at an 
uncomfortable and, for them, unusual pace, and permits the computation of reading latencies for each word in a text. This last point is especially important for the present purposes because it allows us to determine when each of the contextual constraints exert their influence on the target sentence. Moreover, we eliminated the possibility that our sample adopted special metaphor or sarcasm reading strategies (see, Steen, 1993) by intermixing the critical experimental passages amongst a large number of filler passages to read. The critical experimental passages were very similar to those employed in Katz and Pexman (1997) with the major alteration being the addition of text after the target statement, allowing us to investigate contextual effects that arise after the target has been read.

The reading time (RT) data were very clear. Averaged over all conditions, there was little variability in reading the target sentence until the last word in the sentence, which was read 170 msecs. more slowly than the preceding words. This increase in RT at sentence end is a standard finding and is known as sentence wrap-up in the language processing literature, taken as indicating "...A search for referents that have not been assigned, the construction of interclause relations ... and an attempt to handle any inconsistencies that could not be resolved within the sentence" (Just \& Carpenter, 1980, p. 345). Given that most theories of metaphor processing put the processing load for comprehension with the vehicle, in our case the last word, variability in RT for the last word in the sentence should depend on how readily the last word is integrated into the representation of text. If the processing of metaphor in context occurs directly it should be expected that the resolution of any ambiguity would be completed by sentence end and not spillover to the RTs taken downstream (as evidenced by longer RTs taken to start reading the sentence following the target, or even the first few words of the following sentence). Thus, we were most interested to see if spill over occurred, and if such occurrence varied with the contextual constraints.

There was clear evidence that the constraints came into play quite early in the processing sequence. First, on comparing the neutral conditions involving the two neutral conditions in which speaker's occupation (high-metaphor or high- irony) was mentioned with that which there was no mention of occupation, we found reliably longer RTs at the last word of the ambiguous statement for the two conditions involving occupational mention; this effect was most evident for statements that were unfamiliar. Taken together these data indicate that the effect of a social constraints and indicant of speaker's intent, is available at an early stage of statement processing; the familiarity effect suggests that at these early stages of processing the cue is mainly useful in the comprehension of unfamiliar statements, presumably those without a well-established canonical (or salient) meaning. 
Second, there were clear effects based on the nature of the discourse that preceded the target statement. Targets that were in the metaphor-inviting context produced much less spill-over to locations downstream than the same items placed in a sarcasm-inviting condition. In fact the RTs in the two conditions were the same at the last word in the target location, suggesting that regardless of the nature of the context, the metaphoric interpretation of the statement was largely completed by statements end. However, the time taken to proceed to the first word of the sentence following the target was slower (by $54 \mathrm{msec}$ ) in the sarcasm- inviting condition. These data suggest that although the metaphoric content of the statement might be available early, the recognition of the sarcastic commentary takes longer to develop.

Third, there was an interactive etfect of discourse context and occupational mention: when the target statement was uttered by a person from a high-metaphor occupation, RTs at the last word in the statement were substantially slower in the neutral-context condition (by over $100 \mathrm{msecs}$ ) than either the irony-inviting or metaphor-inviting contexts, which did not differ from one another. When the target is uttered by a person from a high-irony occupation, once again we find slower RTs in the neutral-context condition relative to the other two conditions. However, now there are additional effects, with differences found in reading times beyond the statement itself: the time taken to proceed to the next sentence is faster in the metaphor-inviting condition but slower in the irony-inviting condition, a difference of over 100 msecs.

Taken together, the effects can be taken to show that providing enriched context leads to some resolution of meaning of an ambiguous statement by the last word of that statement. Given that in our case, the statements are in the canonical form of a nominal metaphor, these data are consistent with the claim that, with contextual support, metaphoric meaning is available very early in the processing sequence. Second, when the statement is uttered by a person stereotypically understood as a high metaphor user further analysis of the statement does not appear to occur (as evidenced by a failure to find marked spill over effects in reading time beyond the sentence itself) Third, when the speaker is stereotypically understood as a high- irony user additional processing occurs downstream, almost immediately when the discourse context invites an ironic interpretation and more slowly when the context .just supports the default metaphoric interpretation. Thus it appears that at least one social factor (speaker's occupation) plays an important role in the online reading of nonliteral language. When the discourse context is consistent with the sarcastic interpretation, knowledge of the speaker is assessed early and aids in the integration of the target statements's meaning very shortly after the statements has been read; when there is an incongruity between the sarcastic nature of the speaker and a context that invited a non-sarcastic interpretation, this incongruity appears to be initially ignored, but is noted by the beginning of the next sentence. 


\section{Conclusions}

The data support our contention that social factors (operationalized here by stereotypes about the linguistic habits of people in different occupations) are used in assessing the likelihood that a comment was intended metaphorically or as sarcastic commentary. This factor interacted with the familiarity of the comment (and hence the likelihood that the comment has a salient, socially agreed upon meaning) and the nature of the discourse in which it is embedded. These effects are not artifacts created by asking people to rate the sarcasm level of statements or to recall these statements some time after the statement has been encountered, but rather "work" on-line, during the encounter itself. On one hand, the on-line finding that metaphoric meaning is accessed by the end of the sentence is consistent with a body of literature which indicates that metaphoric meaning is directly available when supported by context. We have shown that the evaluation of the sarcastic nature of the metaphor takes somewhat longer to develop, though still occurs relatively rapidly in the comprehension process. Moreover some information (such as social identity of the speaker) is available very early for usage but the integration of this knowledge interacts with statement familiarity and the nature of the preceding discourse. The claim is not that the only factors of relevance are social identity, familiarity and discourse context and we have pointed out earlier in this paper many other contextual constraints that very well might work on-line as well.

So how should we then conceptualize the underlying processes involved in disambiguating nonliteral language? The traditional approach has been to consider the processing as sequential in nature. Characteristic models of this sort include the standard pragmatic model of Searle and the graded saliency model of Giora. Both of these models make the argument that the processing of certain information (literal meaning; salient meaning) has priority and other information, if processed at all, generally occurs only later (via the inferential steps required to reach the understanding that a statement was intended as a metaphor or as sarcasm). Giora's position is somewhat more complex inasmuch as context can boost the accessibility of non-salient information so that, in principle, the salient and less salient information could both be available early in the processing sequence; she argues, however that the boost to non-salient information is not done at a cost to the salient information which is never processed more slowly than non-salient information. The results presented above, involving very early identification of information useful for disambiguating speaker's intent and the interaction of the this information with other constraints is thus not inconsistent with Giora's position. But they also suggest an alternative explanation, namely that multiple 
sources of information are evaluated and integrated continuously, a model successfully applied to understanding the resolution of syntactic ambiguity (see McRae et al., 1998). I would like to argue here that such a model would be a fruitful addition to how we theorize about the processing of "high-order" forms of language ambiguity, including metaphor and sarcasm.

\section{References}

Blasko, D. \& Connine, C. (1993). LtJects of familiarity and aptness on metaphor processing. Journal of Experimental Psychology,: Learning, memory and cognition, 19, 295-308.

Gibbs, R. W. (1986). On the psycholinguistics of sarcasm. Journal of Experimental Psychology: General, I 15, 3-15.

Gibbs (2000). Irony in talk among friends. Metaphor and Symbol, 15, 5-21.

Giora, R., Fein, O. \& Schwartz, T (1998). Irony: graded salience and indirect negation. Metaphor and Symbol, 13, 83-101.

Glucksberg, S. (1991). Beyond literal meanings: The psychology of allusion. Psychological Science, 2, 146-152.

Holtgraves, T. (1991). Interpreting cluestions and replies: effects of face-threat, question form, and gender. Social Psychology Quarterly, 54, I 5-24.

Holtgraves, T. (1992). Interpersonal underpinnings of request strategies: General principles and differences due to culture and gender. Journal of Personality and Social Psychology, 62, 246-256.

Holtgraves, T. (1994). Communication in context: effects of speaker status on the comprehension of indirect requests. Journal of Experimental Psychology,: Learning, memory and cognition, 20, 1205-1218.

Holtgraves, T. (1997a). Politeness and memory for wording of remarks. Memory and Cognition, 25, 106-1 16.

Holtgraves, T. (1997b). Styles of language use: Individual and cultural variability in conversational indirectness. Journal of Personality and Social Psychology, 73, 624-637.

Jorgensen, J. (1996). The functions of sarcastic irony in speech. Journal of Pragmatics, 26, 613634.

Just, M. \& Carpenter, P. (1980). A theory of reading: from eye fixation to comprehension. Psychological review, 87, 329-354

Katz, A. (1996a). On interpreting statements as metaphor or irony: Contextual heuristics and cognitive consequences. In J.S. Mio and A.N. Katz (eds) Metaphor: implications and applications (pp. 1-22). Mahwah, NJ: Lawrence Erlbaum Associates, inc.

Katz, A. (1996b). Experimental Psycholinguistics and figurative language: Circa 1995. Metaphor and Symbolic Activity, 1 1, 17-37.

Katz, A. \& Lee, C. (1993). The role of authorial intent in determining verbal irony and metaphor. Metaphor and Symbolic Activity, H. 257-279. 
Katz, A. \& Pexman, P. (1997). Interpreting figurative statements: Speaker occupation can change metaphor to irony. Metaphor and Symbol, 12, 19-^11.

Kay, P. (1997). Words and the grammar of context. Stanford, CA: CSLI Publications. Kelly, S., Barr, D., Church, R., \& lynch, K. (1999) Offering a hand to pragmatic

understanding: the role of speech and gesture in comprehension and memory. Journal of Memory and Language, 40, 577-592.

Kemper, S. \& Thissen, D. (1981). Memo y for dimensions of requests. Journal of Verbal Learning and verbal Behavior, 20, 552-563

Kreuz, R. (1996). The use of verbal irony: Cues and constraints. In J.S. Mio and A.N. Katz (eds) Metaphor: implications and applications (pp. 23-38). Mahwah, NJ: Lawrence Erlbaum Associates, inc.

Kreuz, R., Kassler, M., C.oppenrath, L \& Allen, B. (in press). Tag questions and common ground effects in the perception of verbal ir ny. Journal of Pragmatics.

Kreuz, R. \& Glucksberg, S. (1989) How to be sarcastic: The echoic reminder theory of verbal irony. Journal of Experimental Psychology: General, 118, 374-386.

Lee, C. \& Katz, A. (1998). The ditferential role of ridicule in sarcasm and irony. Metaphor and Symbol, 13, 1-5.

MacDonald (2000). Neuropsychological studies of sarcasm, Metaphor and Symbol, 15, 85-98. McRae, K,, Spivey-Knowlton, M. K Tanenhaus, M. (1998). Modeling the influence of thematic fit (and other constraints)in on-line sentence comprehension. Journal of Memory and Language, 38, 283-312.

Pexman, P, Ferretti, T. \& Katz, A (2000). Discourse factors that influence on-line reading of metaphor and irony. Discourse process, 29, 201-222.

Pynte, J., Besson, M. Robichon, F-H \& Poli, J. (1996). The time course of metaphor comprehension: An event-related potential study. Brain and Language, 1996, 55, 293 316.

Steen, G. (1993). Metaphor and literary reception. London: Longman Group.

Toplak, M. \& Katz, A. (2000). On the uses of sarcastic irony, Journal of Pragmatics, 32, 1467-1488.

Turner, N. \& Katz, A. (1997). The availability of conventional and of literal meaning during the comprehension of proverbs. Pragmatics and Cognition, 5, 199-133.

Searle, J. (1979). Metaphor. In A Ortony (ed) Metaphor and Thought (pp. 92-123). Cambridge, GB: Cambridge University press.

Vu, H., Kellas, G. \& Paul, S. (in press). Sources of sentence constraint on lexical ambiguity resolution. Memory and Cognition.

Winner, E. (1988). The point of words Children's understanding of metaphor and irony. Cambridge, MA: Harvard University Press. 